\title{
Modulation of the Microbiome in Parkinson's Disease: Diet, Drug, Stool Transplant, and Beyond
}

\author{
Ethan G. Brown ${ }^{1}$ (I) $\cdot$ Samuel M. Goldman ${ }^{1,2}$ \\ Accepted: 28 September 2020 / Published online: 9 October 2020 \\ (C) The American Society for Experimental NeuroTherapeutics, Inc. 2020
}

\begin{abstract}
The gastrointestinal microbiome is altered in Parkinson's disease and likely plays a key role in its pathophysiology, affecting symptoms and response to therapy and perhaps modifying progression or even disease initiation. Gut dysbiosis therefore has a significant potential as a therapeutic target in Parkinson's disease, a condition elusive to disease-modifying therapy thus far. The gastrointestinal environment hosts a complex ecology, and efforts to modulate the relative abundance or function of established microorganisms are still in their infancy. Still, these techniques are being rapidly developed and have important implications for our understanding of Parkinson's disease. Currently, modulation of the microbiome can be achieved through non-pharmacologic means such as diet, pharmacologically through probiotic, prebiotic, or antibiotic use and procedurally through fecal transplant. Novel techniques being explored include the use of small molecules or genetically engineered organisms, with vast potential. Here, we review how some of these approaches have been used to date, important areas of ongoing research, and how microbiome modulation may play a role in the clinical management of Parkinson's disease in the future.
\end{abstract}

Key Words Gastrointestinal microbiome $\cdot$ dysbiosis $\cdot$ Parkinson's disease $\cdot$ therapy $\cdot$ diet $\cdot$ probiotics $\cdot$ fecal microbiota transplantation $\cdot$ organisms $\cdot$ genetically modified

\section{Introduction}

Recent studies provide evidence for a critical role of the gastrointestinal (GI) microbiome in Parkinson's disease (PD). While this work has largely evolved over the last decade, the GI tract has long been thought to play a key role in PD. Nearly 20 years ago, Braak and colleagues proposed the gut mucosa as a portal for the initiation of Parkinson's disease [1]. GI symptoms such as constipation are extremely prevalent in PD [2], often occur long before diagnosis [3-5], and are associated with Lewy body pathology [6] and degeneration of the substantia nigra (Sn) [7]. These findings, along with the detection of alpha-synuclein pathology in the GI tract prior to PD diagnosis [8], a possibly reduced risk of PD in those who

Ethan G. Brown

ethan.brown@ucsf.edu

1 Division of Movement Disorders and Neuromodulation, Weill Institute of Neurology, University of California, San Francisco, CA, USA

2 Division of Occupational and Environmental Medicine, University of California, San Francisco, CA, USA previously underwent vagotomy $[9,10]$, and the demonstration of retrograde transport of alpha-synuclein along the vagus nerve into the brain $[11,12]$, increasingly support Braak's hypothesis that PD pathophysiology may actually start in the gut [1].

Made possible by the advent of high-throughput genetic sequencing [13], the microbiome has been identified as a potential mediator of the PD-gut relationship. The term GI microbiome formally refers to the totality of bacterial genomic material within the GI tract, while the term microbiota refers to the organisms themselves [14]. Because most investigative techniques measure the former, in this review, we will exclusively use the term microbiome. Although they may be important to human biology, we will not discuss the GI virome or mycobiome (fungi) as little is known. Numerous recent studies have confirmed that the GI microbiome differs in people with PD compared with healthy controls [15], but whether this dysbiosis underlies or contributes to PD symptomatology or pathophysiology, as opposed to being a consequence of the disease, is still unclear.

Given the accessibility of the gut, the GI microbiome seems to be a natural target for therapeutic intervention. Clarifying our nascent understanding of its relationship with 
PD is essential for identifying proper therapeutic targets and outcomes. Modulation of the microbiome may help further this understanding, as interventional studies can circumvent some of the limitations of observational studies. While there have been few interventional studies of microbiome modulation in people with PD, studies in related conditions and in animal models can help us understand which approaches may be most successful. In this article, we will summarize what existing studies teach us about modulating the microbiome, review efforts to date to modify the microbiome in $\mathrm{PD}$, and discuss potential future approaches to microbiome modulation for therapeutic benefit in PD.

\section{Techniques for Analyzing the Microbiome}

Understanding the study designs, study populations, sample processing, and sample analytic methods is essential when comparing microbiome studies, as each of these may limit comparability. Conflicting results may be due to differences in study participants' diet, geography, disease severity, smoking habits, comorbidities, and medications. For instance, He and colleagues [16] found that disease-specific models of dysbiosis did not generalize even across regions within the same province. Differences in sample collection and processing are also important sources of variability. How quickly a stool sample is processed and whether or not it is frozen may alter bacterial populations [17]. Several techniques for quantifying organism populations are also available, and many of them are novel and rapidly evolving. These include estimating the diversity within a sample (alpha diversity) or between different samples (beta diversity), which targeted identification of particular organisms of interest such as through sequencing bacterial $16 \mathrm{~S}$ ribosomal RNA and untargeted sequencing of an entire sample through shotgun metagenomics $[18,19]$. More recently, analysis of the RNA transcribed, the proteins expressed, and the metabolites produced (metatranscriptomics, metaproteomics, and metabolomics) has sought to more closely estimate organisms' functional effects, which may have more direct implications for human health and disease [19]. Sophisticated bioinformatic techniques have been developed to visualize changes in microbiome populations [19] or to characterize broader phylogenetic group differences [20]. Each of these steps, from DNA extraction to computational methods of data analysis, commonly differs between studies, reducing comparability and potentially leading to bias [21]. Future research should strive to standardize sample collection and processing, adequately consider potential confounders, ensure sufficient statistical power, and use appropriate analytic techniques. These considerations become especially important for microbiome modulation studies in order to address whether or not therapeutic targets are population-specific and how best to measure efficacy.

\section{How the Microbiome Is Altered in Parkinson's Disease}

Differences in technique may also explain many of the conflicting results from investigations into the microbiome changes seen in PD [15]. Despite these discrepancies, characterizing the common themes that have emerged is an important first step to identify factors that may relate to PD pathophysiology and potential therapeutic targets. To date, more than twenty cross-sectional studies have been published [22-42], of which two with longitudinal follow-up [43, 44].

Thus far, all of these studies have detected differences in the abundance of bacterial taxa between people with PD and healthy controls, though some differences may be explained by sex, age, diet, medications, constipation, and other comorbidities [40]. After adjusting for some of these confounders, the most consistent change thus far includes an increased abundance of the genus Akkermansia, from the family Verrucomicrobiaceae [23, 26, 28, 30, 36-38, 40]. This difference was confirmed in a recent meta-analysis [40] and successfully discriminated between PD and healthy controls using a machine learning algorithm in pooled datasets [45]. Small- to moderate-sized studies have reported decreased abundance in Prevotella or the family Prevotellaceae [31, 33, 36-38]. Decreased Prevotella abundance may be associated with more rapid disease progression [43] and is also seen in idiopathic REM sleep behavior disorder, a condition which is considered a manifestation of early PD [25]. However, two large pooled analyses found no or only minimal associations with Prevotella [40, 45]. Increased abundance in Bifidobacteria, or the family Bifidobacteriaceae [22, 26, 32, $33]$, and decreased abundance in Lachnospiraceae [22, 26, 28, 32-34] have also been observed with some consistency [45], though changes in Lachnospiraceae may be affected by constipation [40]. Increases in the family Lactobacillaceae have also been observed [27, 33, 34, 43], though these may be related to catecholamine- $O$-methyltransferase (COMT) inhibitors [40], a common medication for PD. Differences in several other taxa have been reported, though results are often variable.

How these bacteria interact with human physiology is not well established. Prevotella and Lachnospiraceae, which are reduced in the $\mathrm{PD}$ gut, produce short-chain fatty acids (SCFAs) such as propionate and butyrate that may have anti-inflammatory effects in the central nervous system [46]. Unger et al. [37] used gas chromatography to measure the concentration of SCFAs in fecal samples of people with PD. They noted a decrease in absolute concentrations of butyrate, acetate, and propionate and a decrease in relative 
concentration of butyrate, when compared with those of healthy controls [37]. Butyrate, in particular, is thought to play an anti-inflammatory role through modulation of the NF-kB pathway, which has been found to mediate neurodegeneration in animal models of PD $[47,48]$. Prevotella species also play a specific role in mucin synthesis, and their depletion in PD may contribute to increased gut permeability [36].

However, these proposed mechanisms may be overly simplistic. SCFAs, for instance, may have many different physiologic functions. Sampson et al. [49] showed that SCFAs produced by bacteria were disadvantageous in a transgenic mouse model, being necessary and sufficient to promote microglial activation, nigral degeneration, and motor symptoms. Importantly, these were germ-free mice that likely have many other physiologic differences that make comparisons to human microbiome studies challenging. Furthermore, plasma SCFAs may actually be increased in PD relative to controls [50]. Similarly, Akkermansia, present at increased levels in people with PD, actually have anti-inflammatory and epithelial-strengthening properties in other conditions [51]. Overall, which microbial changes are a consequence of preexisting disease, which are compensatory, and which may be etiologically related are still unclear.

\section{The Role of the Gastrointestinal Tract in PD}

Changes in the PD microbiome may create a more proinflammatory environment through reduced production of SCFAs, direct intestinal immunomodulation, reduction of mucin synthesis, and increased GI permeability. A link between GI inflammation and PD has been suggested on a population level, as inflammatory bowel disease (IBD) has been associated with PD in some [52-55] though not all [56] population-based studies. Higher gut permeability is found in people with PD and is associated with submucosal invasion of Escherichia coli and alpha-synuclein aggregation [57]. This permeability may arise from toll-like receptor 4 (TLR4)-mediated inflammatory signaling, as a higher number of TLR4 and $\mathrm{CD} 3^{+} \mathrm{T}$ cells, and a lower number of tight junction proteins, were found in mucosal biopsies of people with PD compared with healthy controls [58]. Several other inflammatory mediators are over-expressed in colonic biopsies and stool of people with PD, including TNF- $\alpha$, IFN- $\gamma$, VEGF, IL- $1 \alpha$, IL- $1 \beta$, COX-2, and CRP [39, 59-64]. Fecal calprotectin, a marker of inflammation in the stool, is increased in people with PD and correlates with changes in the microbiome [39]. This inflammation and gut permeability may increase exposure to lipopolysaccharide (LPS), a constituent of gram-negative bacterial membranes used in animal models to induce nigral degeneration [65]. The direction of causality for these observations is not established: increased expression of alphasynuclein in the substantia nigra can induce inflammation in the GI tract and produce changes in the microbiome [66]. On the other hand, a "bottom-up" approach has also been demonstrated, as transplanted stool from patients with PD produces motor deficits in transgenic mice [49].

If the microbiome alterations observed in $\mathrm{PD}$ are indeed causally related to disease, they may act in a variety of different ways. One hypothesis posits that gut dysbiosis directly leads to neurodegeneration through induction of alphasynuclein aggregation and trans-vagal spread of the protein to the dorsal motor nucleus of the vagus and into the brain [1]. Indeed, orally exposing rats to bacteria that produce specific types of amyloid protein can induce alpha-synuclein aggregation and inflammation in the gut and brain [67]. Furthermore, TLR4 knockout mice given oral rotenone were protected from gastrointestinal inflammation, myenteric alpha-synuclein aggregation, substantia nigra degeneration, and motor dysfunction, but not microbiome changes, suggesting that this inflammatory cascade is necessary for GI changes to influence neuronal damage [58]. Alternatively, dysbiosis may increase peripheral inflammation, which may either induce neurotoxicity or exacerbate ongoing neurodegeneration. Evidence of increased peripheral inflammation in PD has been found in several studies $[68,69]$. Finally, inflammation from dysbiosis may act locally, worsening gastrointestinal symptoms of PD or impairing medication absorption.

As we consider modulation of the microbiome, all of these potential roles must be kept in mind. The mechanism of microbiome modulation chosen, as well as the subset of people with PD likely to benefit, may depend on specific goalsfor example, symptomatic therapy or disease progression. Multiple techniques to modulate the microbiome have been studied and will be reviewed here, including diet, pharmacologic therapies, and procedures.

\section{Mechanisms of Modulation}

\section{Diet}

Perhaps the most effective way to substantially and persistently alter the microbiome is through diet [70]. Many different aspects of the diet, from the amount and frequency of eating to components such as dairy, breads, and soft drinks, contribute to inter- and intra-individual variations of the microbiome [71]. In addition, numerous dietary nutrients and supplements have been evaluated in animal and human clinical studies and found to alter populations of beneficial bacteria [72]. In mice, as much as $57 \%$ of inter-individual variability in the microbiome may be explained by dietary factors [73]. Changes in diet can lead to changes in the microbiome within a day [74], with a new steady-state bacterial community achieved within several days [75]. In the elderly, differences in the microbiome mirrored differences in diet and were able 
to distinguish between community-dwelling persons and residents of institutional or long-term care facilities; these differences were also related to measures of frailty and functional independence [76]. Dietary modulation of the microbiome is therefore already recommended as a way to manage many conditions such as metabolic syndrome and inflammatory bowel disease [77].

Although findings are highly inconsistent, observational studies have found associations of dietary habits and the risk of PD. Higher adherence to "plant-based" diets, such as the Mediterranean or MIND diet, as opposed to Western-style, higher-meat-and-fat diets, has been associated with a lower risk of developing PD and a later age of onset [78-80]. In a large prospective cohort of elderly adults without parkinsonism at baseline, parkinsonian motor symptoms were less likely to develop and progressed more slowly in those adherent to the MIND diet [81]. Similarly, the probability of having prodromal PD as defined by the International Parkinson and Movement Disorder Society [82] was found to be lower in persons who adhered to a Mediterranean diet [83].

Several caveats of diet-focused observational study designs temper the interpretation of these studies. A largely plantbased, high-fiber diet has many cardiovascular and other health benefits that may confound its relationship with PD. In addition, diet studies are notoriously difficult to interpret as patients willing to adhere to diets may be generally healthier and also maintain other elements of a healthy lifestyle, such as exercise, which may be associated with a reduced risk of PD $[84,85]$.

Nonetheless, dietary modifications can result in changes to the same microbiome genera that are altered in PD. For instance, a long-term plant-based diet is associated more often with Prevotella [86], and the abundance of Prevotella increases or decreases with changes in dietary fiber [74, 87]. Paralleling these observations, changes in dietary fiber are associated with subsequent changes in production of SCFAs including butyrate [88]. Interestingly, the level of SCFAs generated by intake of a particular dietary component depends on an individual's microbiome. Furthermore, in a mouse model, reducing fiber leads to metabolism of the GI mucus layer, breakdown of the GI epithelium, and increased inflammation [89], similar to the mechanistic theory of dysbiosis in PD [90].

Dietary interventions may therefore be an effective method for altering the microbiome, improving symptoms, or even modifying progression in PD. As mentioned above, a Mediterranean-style, high-fiber diet has significant scientific rationale. In a large cohort of elderly participants, adherence to the Mediterranean diet correlated with increased SCFAproducing bacteria, reduced frailty (including faster gait speed), and reduced peripheral inflammatory markers [91]. Although a Mediterranean dietary intervention has not been directly studied in PD, a small trial implemented a 14-day ovo-lacto vegetarian diet that included foods with high
SCFA content in a cohort of sixteen people with PD [92]. Ten participants received an additional intervention of a daily rectal enema for 8 days. There were no significant changes in the microbiome before and after intervention in the diet-only group. However, the group that received the enemas as well as the dietary intervention had a reduction in Clostridiaceae, part of the putatively inflammatory family Clostridia which have been found to be increased in PD [93]. One year after the intervention, UPDRS-III scores had decreased significantly in both the diet-only and the diet-enema groups [92]. Notably, medication requirements decreased in the dietenema subgroup but increased in the diet-only group. Though limited by small sample size and lack of a control group, this suggests that dietary interventions in PD aimed at microbiome manipulation are feasible and worth future study.

Other dietary interventions have been shown to improve symptoms and possibly slow progression in $\mathrm{PD}$, though studies are small, brief, and minimally controlled. The ketogenic diet, a low-carbohydrate, high-fat diet thought to increase ketosis, may improve mitochondrial respiration in the substantia nigra and thereby slow neurodegeneration [94]. In one small study, 1 month of a ketogenic diet improved motor scores in people with PD [95]. In another more recent study, both the ketogenic diet and a low-fat diet improved motor and nonmotor symptoms of people with PD, though non-motor symptoms improved to a greater extent in those following the ketogenic diet [96]. Both of these diets may lead to beneficial changes in the microbiome through increased fiber, but the specific effects on the microbiome in these patients were not evaluated.

Another important aspect of diet that may beneficially influence the microbiome is caffeine use, which is associated with a reduced risk of PD [97]. Coffee seems to have beneficial effects on the microbiome, including greater diversity [98], a higher abundance of Bacteroides and Prevotella [99], and a higher abundance of Bifidobacterium [100], bacteria associated with decreased gastrointestinal inflammation and permeability [36]. In addition to caffeine, these microbiome changes may be explained by coffee's dietary fiber and polyphenols [99] or by improving constipation through increased colonic motility [101]. Thus far, no PD studies have directly examined microbiome changes after caffeine use, though one small trial failed to show symptomatic motor benefit after caffeine administration [102]. Careful dietary histories and incorporation of caffeine metabolites [103] into microbiome studies will further clarify this association.

\section{Probiotics and Prebiotics}

Probiotics are isolated, live microorganisms, often formulated into capsules, tablets, or powders, that can be administered for health purposes [104]. Probiotics often include Bifidobacterium and Lactobacillus, two strains that have been studied most 
frequently for their health benefit, though they may involve other lactic acid-producing bacteria or other types of yeasts [104]. Prebiotics are dietary ingredients that are not alive but induce changes in the composition or activity of GI flora [105]. These ingredients are often not digested by the host and remain in the GI tract. Both of these agents have already shown extensive clinical benefit in GI diseases, which includes preventing antibiotic-associated diarrhea, decreasing recurrence of Clostridium difficile infection, and maintaining remission in inflammatory bowel disease [106].

Indeed, GI symptomatic benefits of probiotic administration have been suggested in PD. In a recent trial, 120 people with PD were randomized to consume either a fermented milk consisting of multiple probiotic strains and prebiotic fiber or placebo once daily for 4 weeks [107]. During the last 2 weeks of the intervention, the group receiving the fermented milk had a higher number of complete bowel movements and a greater decrease in the use of other laxatives compared with the placebo group [107]. Importantly, rather than directly modifying the microbiome, the presence of dietary fiber in the intervention, known to increase stool frequency [108], could also have explained the improvement in constipation. Probiotic use helps alleviate constipation in other populations [109], though the generalizability to PD is unclear as the cause of constipation may be different. The mechanisms responsible for the beneficial effects of probiotics are still unclear but may include direct effects on gut motility, increases in mucin secretion, or immunomodulatory interactions [110].

Beyond their direct GI effects, probiotics also have potential disease-modifying benefits through microbiome modulation. Notably, probiotics are not thought to simply replace existing microbial communities with "healthier" species. Microbial ecosystems are extremely complex. Many species cannot successfully be administered orally, and even when they can, the existing dominant species may not be easily displaced. However, probiotic administration may inhibit the growth of pathogenic bacteria, improve the production and absorption of vitamins and minerals, and modulate GI inflammation [111]. In two toxin-induced mouse models of PD, an orally administered probiotic mixture consisting of Lactobacillus and Bifidobacterium reduced degeneration of dopaminergic cells in the substantia nigra and led to less severe motor decline [112]. These changes seemed to be mediated by increasing butyrate production, which inhibited nigral inflammation and increased neurotrophic factors [112]. Coculturing a similar mix of probiotic strains with peripheral immune cells (peripheral blood mononuclear cells (PBMCs)) extracted from people with PD decreased cytokine release and free radical production [113]. In another mouse model of PD, daily administration of probiotics comprised of multiple strains of bacteria led to improvement in multiple measures of motor function and reduced degeneration of dopaminergic neurons in the substantia nigra [114]. Similarly, a novel probiotic agent, SLAB51, attenuated nigral degeneration and inflammation and improved motor behavior in another toxin-induced mouse model of PD [115]. In vitro studies suggest that SLAB51 prevents neuronal death through increasing brain-derived neurotrophic factor (BDNF) [115]. Finally, in a Caenorhabditis elegans model, administration of Bacillus subtilis decreased alpha-synuclein aggregation and increased clearance of pre-formed aggregates by altering sphingolipid metabolism and creating a biofilm in the gut [116]. Thus, probiotics have multiple potentially beneficial effects that may act on specific mechanisms in PD pathogenesis.

While this pre-clinical evidence for probiotic benefit in PD is encouraging, human trials are still limited. A recent doubleblind controlled trial randomized 60 people with $\mathrm{PD}$ to receive either a probiotic containing Lactobacillus and Bifidobacterium or placebo for 12 weeks [117]. Motor signs and symptoms as measured by the Movement Disorders Society-Unified Parkinson's Disease Rating Scale (MDS-UPDRS) improved in the probiotic group compared with the placebo group. These improvements corresponded with lower serum levels of C-reactive protein, a marker of systemic inflammation; higher levels of the antioxidant glutathione; and increased insulin sensitivity [117]. In a similar study, expression of the proinflammatory cytokines IL-1, IL-8, and TNF-alpha in PBMCs was reduced after probiotic intake, while expression of the anti-inflammatory regulators TNF-beta and PPARgamma was increased [113]. Notably, changes in constipation were not reported in these studies, which may be an important confounder for symptomatic improvement, and changes in the microbiome were not evaluated. Future large and longer-term studies may inform potential beneficial effects of probiotics in PD and how they affect PD dysbiosis.

\section{Antibiotics}

From a pharmacologic perspective, the most profound way to alter the GI microbiome is through antibiotics. While antibiotics are often prescribed to target particular pathogenic bacteria, their effect on beneficial bacterial species has been recognized for some time [118]. Antibiotic-associated diarrhea is one of the most common "off-target" complications. It is caused by a reduction of bacterial diversity and alteration of physiologic, anti-inflammatory functions of healthy flora, which allows for an overgrowth of deleterious organisms, most commonly $C$. difficile $[119,120]$. How significantly antibiotics change the gut microbiome depends on their spectrum of effect, to what extent they are absorbed systemically, and how they are excreted [121]. For instance, broadspectrum antibiotics that are secreted in bile acid have a profound impact on the colonic microbiome [122]. In addition, while many of these changes resolve over months, in some cases, antibiotic-induced population shifts and microbial 
resistance can be detected years after a short-term administration of antibiotics [122].

Antibiotic use and subsequent dysbiosis may also play a role in PD. A recent population-based study in Finland found an increased risk of developing PD as long as 10-15 years after exposure to certain types of antibiotics, specifically antianaerobics and tetracyclines [123]. An alternative interpretation of this association is that infections may predispose people to PD, or that people with early undiagnosed PD may be more likely to require antibiotics. However, the authors argue that the association would not be dependent on the type of antibiotic in that case. Furthermore, they did not see a significant relationship between hospital diagnoses of infections and subsequent PD [123]. Thus, microbiome changes due to antibiotics might potentially contribute to neurologic disease years afterwards. Another recent study in the USA did not find a relationship between antibiotic use and PD risk, though this study was limited by reliance on a survey where respondents had to recall their antibiotic use in the past [124].

Importantly, not all antibiotic-induced microbiome changes are harmful. Antibiotic administration has been found to improve the course of many non-infectious conditions where dysbiosis is thought to be an underlying cause, including IBD and irritable bowel syndrome (IBS) [120]. In PD, antibiotic administration in some dysbiotic conditions may improve not just GI symptoms but also symptoms of PD. For example, Helicobacter pylori eradication was found to reduce gastritis, improve levodopa pharmacokinetics, and prolong the time of levodopa's clinical efficacy in a placebo-controlled trial [125]. A more recent, larger trial did not find a similarly beneficial effect, though the trial was designed to study the efficacy of levodopa as opposed to its duration of action [126]. In a small, open-label study of patients with small intestinal bacterial overgrowth (SIBO), rifaximin use was associated with fewer motor fluctuations and longer therapeutic benefit of PD medications [127], though these results have not been replicated in a placebo-controlled trial. Notably, rifaximin has low systemic absorption, suggesting that any clinical benefit comes from intra-intestinal mechanisms. While rifaximin does not seem to alter the overall abundance of GI bacteria as significantly as other antibiotics, it may ultimately promote bacteria with anti-inflammatory properties such as Lactobacillus, demonstrating how a targeted antibiotic could potentially induce a healthier microbiome population [128].

Whether antibiotic therapy might slow PD progression or improve symptoms more generally is still unclear. Minocycline, a tetracycline antibiotic, showed neuroprotective effects in a PD mouse model [129]. This evidence led to a phase 2 , randomized, controlled trial which unfortunately did not show benefit [130]. Notably, its efficacy in the mouse model may have been due to direct systemic antioxidant and anti-inflammatory effects, rather than effects on the gut or the microbiome [131]. The inability to separate intra-intestinal effects from the systemic effects of many antibiotic agents, as well as their unpredictable impact on GI ecology, is a significant impediment to their use for targeted microbiome manipulation. Whether there is a therapeutic role for antibioticmediated regulation of the microbiome in PD remains to be determined.

\section{Stool Transplant}

Fecal microbiome transplant (FMT), or microbial replacement therapy, is increasingly used and proposed as an effective and safe method for restoring a healthy intestinal microbiome. FMT involves transferring of fecal material from a healthy donor to a patient with dysbiosis. Transfer can occur through different mechanisms, including orally administered capsules, gastroduodenal endoscopy, colonoscopy, or enema. Successful repopulation of gut flora varies considerably by method, with colonoscopy being most efficacious [132].

From a clinical standpoint, FMT has been shown to be highly effective in otherwise refractory $C$. difficile infections, and investigations into other types of GI and non-GI conditions are rapidly increasing [133]. For instance, several randomized, controlled trials of FMT have targeted obesity and metabolic syndrome, with some showing at least short-term improvement in insulin sensitivity and glycemic index [134]. A recent placebo-controlled trial in obese patients showed sustained alterations in microbiota after oral FMT administration from a lean donor, though body mass index (BMI) did not change [135]. FMT has been preliminarily explored in several neurologic conditions, though to a much smaller extent [136]. Clinical benefit of FMT has been reported in autism spectrum disorder, Tourette's syndrome, epilepsy, diabetic neuropathy, and multiple sclerosis, but largely in the form of case reports or case series [136].

Support for the use of FMT in PD is mostly driven by animal models. As noted above, transgenic mice that received stool transplants from people with PD manifested worsening of motor function compared with mice that received stool from healthy controls [49]. In a separate experiment, Sun et al. [137] transplanted feces from healthy mice to mice that had received MPTP, a toxin that causes degeneration of substantia nigral dopaminergic neurons and Parkinson-like features. After receiving the healthy donor fecal transplant, the MPTP mice showed improved GI dysbiosis, higher levels of striatal dopamine, less nigral degeneration, and improved motor function [137]. These changes were thought to be mediated by changes in SCFAs and reduced inflammatory mediators in the MPTP mice [137].

The literature on the effects of FMT in people with PD is still limited. A case report describes a patient with PD and medically refractory constipation who received FMT via colonoscopy, with subsequent resolution of constipation [138]. Notably, by 1 week after transplant, the patient's motor 
symptoms had improved significantly, though they began worsening again and were back to baseline values 3 months after FMT [138]. This improvement may have been due to a placebo effect, or simply from resolution of constipation and discomfort, which can often improve motor symptoms of PD. On the other hand, the microbiome of the patient most closely resembled that of the donor at 1 week after transplant, before drifting back over time to its prior composition [138]. More recently, a small, open-label study of FMT delivered either through a nasojejunal or colonic route was conducted in people with PD who were not receiving adequate benefit from dopaminergic medications [139]. Participants showed improvement in both motor and non-motor (e.g., anxiety, depression) scores at 1 month and 3 months after the intervention, though stratified analysis revealed improvement only after colonic FMT. The strong placebo response in PD again makes these results challenging to interpret, supporting the need for randomized, controlled trials (e.g., NCT03808389) to clarify the effect of FMT on the microbiome and clinical symptoms in PD.

Overall, our understanding of the therapeutic application of FMT is still in its earliest stages. The screening, processing, and administration of donor material are quite heterogenous between institutions, challenging the interpretation of efficacy and safety data [132]. New safety concerns regarding screening procedures recently arose after six people treated with FMT from the same stool bank developed pathogenic E. coli infections; four of the six were hospitalized, and two patients died $\{\mathrm{FDA}\}$. Furthermore, while identifying a healthy donor may be straightforward in the treatment of $C$. difficile infections, the criteria are less clear when considering FMT as a therapeutic intervention for neurodegenerative disease. For instance, family members are a popular source of FMT donations in clinical practice, but in the case of PD, shared genes and environment may result in microbiomes that might not convey any benefit [140].

\section{Targeted Drug/Small Molecule}

As we learn more about the nuances of the microbiome and how it interacts with host physiology, the non-specific modalities described above may become less applicable. More and more targeted therapies are being designed that are not only disease-specific but also address specific disease-related morbidities. Because each individual's microbiome is a unique and highly evolved system, the approach to its modulation may involve novel therapeutics and a more personalized approach than is typically used to treat other organ system dysfunctions.

For instance, in PD, GI flora have long been thought to play a role in the metabolism of levodopa, the most effective PD pharmacologic treatment $[141,142]$. Greater GI metabolism would theoretically reduce the amount of levodopa available to the brain, which could contribute to the dose failures often seen in advanced PD [143]. Recently, two reports have shown that several gut bacterial species do indeed harbor a tyrosine decarboxylase (TyrDC) enzyme that can metabolize levodopa $[143,144]$. These bacteria reside in the small intestine where levodopa is metabolized, are found in the feces of people with $\mathrm{PD}$, and correlate with levodopa dose and disease duration [143]. Furthermore, they are not inhibited by carbidopa, a drug co-administered with levodopa to block its peripheral metabolism [144]. However, $S$-alpha-fluoromethyltyrosine (AFMT), an amino acid that inhibits bacterial TyrDC, increased serum levels of levodopa in mice when coadministered [144]. While the clinical relevance of this observation is unknown, it demonstrates the potential of targeting a very specific function of the microbiome for therapeutic benefit.

Synthetic biology, the field of genetically engineering microorganisms for specific purpose, also shows significant potential for microbiome modulation. Investigated predominantly in animal models, synthetic bacteria may be engineered to target specific pathogens, facilitate GI immunomodulation, diagnose occult conditions, and deliver medications that are challenging to administer systemically $[145,146]$. Furthermore, the potential benefit of synthetic biology lies not just in the targeted nature of the therapeutic but also in the opportunity for sensing mechanisms that can adjust the delivery of therapy according to particular environments [146]. In a recent example, synthetic biologic techniques were used to develop a potential treatment for the genetic metabolic disorder phenylketonuria (PKU), which is caused by insufficient or defective phenylalanine hydroxylase enzyme. Unless they adhere to a strict lifetime diet low in the amino acid phenylalanine, persons with PKU develop severe and irreversible psychiatric and neurologic features. Isabella and colleagues [147] developed an engineered $E$. coli strain that expresses phenylalanine-metabolizing enzymes under anoxic conditions. Administered orally, the engineered bacteria colonize the gut and degrade dietary phenylalanine, thereby reducing its buildup in the serum. A promising phase 1/2a trial in humans was recently completed. Though few examples have reached human trials, this technology has significant potential to address a range of human disorders through targeted modulation of the gut microbiota [146]. Before such technology becomes applicable in PD, however, a more consistent profile of metabolic abnormalities resulting from dysbiosis is essential.

\section{Conclusions}

Clarifying the role of the microbiome in PD will enable identification of therapeutic targets in a relatively accessible physiologic system. The microbiome may affect disease causation, 
progression, symptomatology, and response to therapy. Whether an individual's microbiome imparts an advantageous or disadvantageous environment likely varies between each of these disease stages, as well as between individuals. Nonetheless, some common themes have emerged. The gutbrain axis is clearly important. Peripheral inflammation begets systemic and, possibly, CNS inflammation, and a proinflammatory microbiome is likely deleterious or even causally related. Modulation of the gut microbiome to establish a less inflammatory environment or produce more short-chain fatty acids may be an important intervention. Probiotics and prebiotics hold promise in this regard. Persons with relatively advanced disease whose microbiomes may have been selected for levodopa-metabolizing species may benefit from antibiotic therapy or targeted synthetic approaches.

Modulating the microbiome directly will also contribute to our understanding of its deleterious and protective characteristics, bypassing the many limitations of the aforementioned observational and animal studies. But, modulating the microbiome is an incredibly complicated proposal and not without risk. One major obstacle, similar to other aspects of PD research, is the lack of a consistent microbiome biomarker that can identify pathology and be used to track treatment response. Standardized methods of microbiome analysis and increased attention to patient selection will be essential for establishing such a biomarker. Studies also should be designed with specific clinical targets in mind, such as gastrointestinal symptoms or PD-related symptoms. Even apparently straightforward symptoms may have multiple mechanisms. For example, a recent trial failed in part because of inadequate criteria for constipation, which may have different characteristics in PD than in other conditions [148]. Careful patient selection and biomarker follow-up will be particularly important in microbiome studies that address disease modification, as even the definition of PD progression is highly variable [149]. Furthermore, the durability of therapeutic benefit of these interventions is still unknown, and an objective method to monitor follow-up will be essential to distinguish between re-constitution of dysbiosis and progression of underlying disease. Overall, more randomized, controlled trials are needed, with clearly defined inclusion criteria, specific microbiome targets, and reliably measured endpoints.

In general, the tools we currently have to modulate the microbiome are somewhat blunt, inducing broad and often poorly understood or predictable changes in a complex ecology. In the future, more specific therapeutic techniques may target not only particular aspects of the microbiome but also specific subgroups of conditions, allowing for truly personalized medicine.

The extent of the GI microbiome's impact on PD still has to be clarified, but the evidence thus far is encouraging that microbiome modulation will 1 day be an effective approach for treatment and, potentially, for prevention.
Required Author Forms Disclosure forms provided by the authors are available with the online version of this article.

\section{References}

1. Braak H, Rub U, Gai WP, Del Tredici K. Idiopathic Parkinson's disease: possible routes by which vulnerable neuronal types may be subject to neuroinvasion by an unknown pathogen. J Neural Transm (Vienna). 2003;110(5):517-36.

2. Pfeiffer RF. Gastrointestinal dysfunction in Parkinson's disease. Parkinsonism Relat Disord. 2011;17(1):10-5.

3. Abbott RD, Petrovitch H, White LR, Masaki KH, Tanner CM, Curb JD, et al. Frequency of Bowel Movements and the Future Risk of Parkinson's Disease. Neurology. 2001;57(3):456-62.

4. Savica R, Carlin JM, Grossardt BR, Bower JH, Ahlskog JE, Maraganore DM, et al. Medical records documentation of constipation preceding Parkinson disease: A case-control study. Neurology. 2009;73(21):1752-8.

5. Gao X, Chen H, Schwarzschild MA, Ascherio A. A Prospective Study of Bowel Movement Frequency and Risk of Parkinson's Disease. American Journal of Epidemiology. 2011;174(5):54651.

6. Abbott RD, Ross GW, Petrovitch H, Tanner CM, Davis DG, Masaki $\mathrm{KH}$, et al. Bowel movement frequency in late-life and incidental Lewy bodies. Movement Disorders. 2007;22(11): 1581-6.

7. Petrovitch H, Abbott RD, Ross GW, Nelson J, Masaki KH, Tanner CM, et al. Bowel movement frequency in late-life and substantia nigra neuron density at death. Mov Disord. 2009;24(3):371-6.

8. Stokholm MG, Danielsen EH, Hamilton-Dutoit SJ, Borghammer P. Pathological alpha-synuclein in gastrointestinal tissues from prodromal Parkinson disease patients. Ann Neurol. 2016;79(6): 940-9.

9. Svensson E, Horvath-Puho E, Thomsen RW, Djurhuus JC, Pedersen L, Borghammer P, et al. Vagotomy and subsequent risk of Parkinson's disease. Ann Neurol. 2015;78(4):522-9.

10. Liu B, Fang F, Pedersen NL, Tillander A, Ludvigsson JF, Ekbom A, et al. Vagotomy and Parkinson disease. Neurology. 2017;88(21):1996.

11. Kim S, Kwon SH, Kam TI, Panicker N, Karuppagounder SS, Lee $\mathrm{S}$, et al. Transneuronal Propagation of Pathologic alpha-Synuclein from the Gut to the Brain Models Parkinson's Disease. Neuron. 2019;103(4):627-41 e7.

12. Lohmann S, Bernis ME, Tachu BJ, Ziemski A, Grigoletto J, Tamguney G. Oral and intravenous transmission of alphasynuclein fibrils to mice. Acta Neuropathol. 2019;138(4):515-33.

13. Kuczynski J, Lauber CL, Walters WA, Parfrey LW, Clemente JC, Gevers D, et al. Experimental and analytical tools for studying the human microbiome. Nat Rev Genet. 2011;13(1):47-58.

14. Sender R, Fuchs S, Milo R. Revised Estimates for the Number of Human and Bacteria Cells in the Body. PLoS Biol. 2016;14(8): e1002533.

15. Boertien JM, Pereira PAB, Aho VTE, Scheperjans F. Increasing Comparability and Utility of Gut Microbiome Studies in Parkinson's Disease: A Systematic Review. J Parkinsons Dis. 2019;9(s2):S297-S312.

16. He Y, Wu W, Zheng HM, Li P, McDonald D, Sheng HF, et al. Regional variation limits applications of healthy gut microbiome reference ranges and disease models. Nat Med. 2018;24(10):15325 . 
17. Tedjo DI, Jonkers DM, Savelkoul PH, Masclee AA, van Best N, Pierik MJ, et al. The effect of sampling and storage on the fecal microbiota composition in healthy and diseased subjects. PLoS One. 2015;10(5):e0126685.

18. Fraher MH, O'Toole PW, Quigley EM. Techniques used to characterize the gut microbiota: a guide for the clinician. Nat Rev Gastroenterol Hepatol. 2012;9(6):312-22.

19. Galloway-Peña J, Hanson B. Tools for Analysis of the Microbiome. Digestive Diseases and Sciences. 2020;65(3):67485 .

20. Washburne AD, Morton JT, Sanders J, McDonald D, Zhu Q, Oliverio AM, et al. Methods for phylogenetic analysis of microbiome data. Nature Microbiology. 2018;3(6):652-61.

21. Poussin C, Sierro N, Boué S, Battey J, Scotti E, Belcastro V, et al. Interrogating the microbiome: experimental and computational considerations in support of study reproducibility. Drug Discovery Today. 2018;23(9):1644-57.

22. Barichella M, Severgnini M, Cilia R, Cassani E, Bolliri C, Caronni $\mathrm{S}$, et al. Unraveling gut microbiota in Parkinson's disease and atypical parkinsonism. Mov Disord. 2019;34(3):396-405.

23. Bedarf JR, Hildebrand F, Coelho LP, Sunagawa S, Bahram M, Goeser F, et al. Functional implications of microbial and viral gut metagenome changes in early stage L-DOPA-naive Parkinson's disease patients. Genome Med. 2017;9(1):39.

24. Hasegawa S, Goto S, Tsuji H, Okuno T, Asahara T, Nomoto K, et al. Intestinal Dysbiosis and Lowered Serum Lipopolysaccharide-Binding Protein in Parkinson's Disease. PLoS One. 2015;10(11):e0142164.

25. Heintz-Buschart A, Pandey U, Wicke T, Sixel-Doring F, Janzen A, Sittig-Wiegand E, et al. The nasal and gut microbiome in Parkinson's disease and idiopathic rapid eye movement sleep behavior disorder. Mov Disord. 2018;33(1):88-98.

26. Hill-Burns EM, Debelius JW, Morton JT, Wissemann WT, Lewis MR, Wallen ZD, et al. Parkinson's disease and Parkinson's disease medications have distinct signatures of the gut microbiome. Mov Disord. 2017;32(5):739-49.

27. Hopfner F, Kunstner A, Muller SH, Kunzel S, Zeuner KE, Margraf NG, et al. Gut microbiota in Parkinson disease in a northern German cohort. Brain Res. 2017;1667:41-5.

28. Keshavarzian A, Green SJ, Engen PA, Voigt RM, Naqib A, Forsyth CB, et al. Colonic bacterial composition in Parkinson's disease. Mov Disord. 2015;30(10):1351-60.

29. Li W, Wu X, Hu X, Wang T, Liang S, Duan Y, et al. Structural changes of gut microbiota in Parkinson's disease and its correlation with clinical features. Sci China Life Sci. 2017;60:1223-33.

30. Li C, Cui L, Yang Y, Miao J, Zhao X, Zhang J, et al. Gut Microbiota Differs Between Parkinson's Disease Patients and Healthy Controls in Northeast China. Front Mol Neurosci. 2019;12:171.

31. Lin CH, Chen CC, Chiang HL, Liou JM, Chang CM, Lu TP, et al. Altered gut microbiota and inflammatory cytokine responses in patients with Parkinson's disease. J Neuroinflammation. 2019;16(1):129.

32. Lin A, Zheng W, He Y, Tang W, Wei X, He R, et al. Gut microbiota in patients with Parkinson's disease in southern China. Parkinsonism Relat Disord. 2018;53:82-8.

33. Petrov VA, Saltykova IV, Zhukova IA, Alifirova VM, Zhukova NG, Dorofeeva YB, et al. Analysis of Gut Microbiota in Patients with Parkinson's Disease. Bull Exp Biol Med. 2017;162(6):734-7.

34. Pietrucci D, Cerroni R, Unida V, Farcomeni A, Pierantozzi M, Mercuri NB, et al. Dysbiosis of gut microbiota in a selected population of Parkinson's patients. Parkinsonism Relat Disord. 2019;65:124-30.

35. Qian Y, Yang X, Xu S, Wu C, Song Y, Qin N, et al. Alteration of the fecal microbiota in Chinese patients with Parkinson's disease. Brain Behav Immun. 2018;70:194-202.
36. Scheperjans F, Aho V, Pereira PA, Koskinen K, Paulin L, Pekkonen E, et al. Gut microbiota are related to Parkinson's disease and clinical phenotype. Mov Disord. 2015;30(3):350-8.

37. Unger MM, Spiegel J, Dillmann KU, Grundmann D, Philippeit H, Burmann J, et al. Short chain fatty acids and gut microbiota differ between patients with Parkinson's disease and age-matched controls. Parkinsonism Relat Disord. 2016;32:66-72.

38. Vidal-Martinez G, Chin B, Camarillo C, Herrera GV, Yang B, Sarosiek I, et al. A Pilot Microbiota Study in Parkinson's Disease Patients versus Control Subjects, and Effects of FTY720 and FTY720-Mitoxy Therapies in Parkinsonian and Multiple System Atrophy Mouse Models. J Parkinsons Dis. 2020;10(1): 185-92.

39. Weis S, Schwiertz A, Unger MM, Becker A, Fassbender K, Ratering S, et al. Effect of Parkinson's disease and related medications on the composition of the fecal bacterial microbiota. NPJ Parkinsons Dis. 2019;5:28.

40. Nishiwaki H, Ito M, Ishida T, Hamaguchi T, Maeda T, Kashihara $\mathrm{K}$, et al. Meta-Analysis of Gut Dysbiosis in Parkinson's Disease. Movement Disorders. 2020.

41. Cirstea MS, Yu AC, Golz E, Sundvick K, Kliger D, Radisavljevic N, et al. Microbiota Composition and Metabolism Are Associated With Gut Function in Parkinson's Disease. Movement Disorders. 2020;35(7):1208-17.

42. Baldini F, Hertel J, Sandt E, Thinnes CC, Neuberger-Castillo L, Pavelka L, et al. Parkinson's disease-associated alterations of the gut microbiome predict disease-relevant changes in metabolic functions. BMC Biol. 2020;18(62).

43. Aho VTE, Pereira PAB, Voutilainen S, Paulin L, Pekkonen E, Auvinen P, et al. Gut microbiota in Parkinson's disease: Temporal stability and relations to disease progression. EBioMedicine. 2019;44:691-707.

44. Minato T, Maeda T, Fujisawa Y, Tsuji H, Nomoto K, Ohno K, et al. Progression of Parkinson's disease is associated with gut dysbiosis: Two-year follow-up study. PLoS One. 2017;12(11): e0187307.

45. Pietrucci D, Teofani A, Unida V, Cerroni R, Biocca S, Stefani A, et al. Can Gut Microbiota Be a Good Predictor for Parkinson's Disease? A Machine Learning Approach. Brain Sci. 2020;10(4).

46. Fung TC, Olson CA, Hsiao EY. Interactions between the microbiota, immune and nervous systems in health and disease. Nat Neurosci. 2017;20(2):145-55.

47. Meijer K, de Vos P, Priebe MG. Butyrate and other short-chain fatty acids as modulators of immunity: what relevance for health? Curr Opin Clin Nutr Metab Care. 2010;13(6):715-21.

48. Flood PM, Qian L, Peterson LJ, Zhang F, Shi JS, Gao HM, et al. Transcriptional Factor NF-kappaB as a Target for Therapy in Parkinson's Disease. Parkinsons Dis. 2011;2011:216298.

49. Sampson TR, Debelius JW, Thron T, Janssen S, Shastri GG, Ilhan $\mathrm{ZE}$, et al. Gut Microbiota Regulate Motor Deficits and Neuroinflammation in a Model of Parkinson's Disease. Cell. 2016;167(6):1469-80 e12.

50. Shin C, Lim Y, Lim H, Ahn TB. Plasma Short-Chain Fatty Acids in Patients With Parkinson's Disease. Mov Disord. 2020.

51. Hiippala K, Jouhten H, Ronkainen A, Hartikainen A, Kainulainen $\mathrm{V}$, Jalanka $J$, et al. The Potential of Gut Commensals in Reinforcing Intestinal Barrier Function and Alleviating Inflammation. Nutrients. 2018;10(8).

52. Weimers P, Halfvarson J, Sachs MC, Saunders-Pullman R, Ludvigsson JF, Peter I, et al. Inflammatory Bowel Disease and Parkinson's Disease: A Nationwide Swedish Cohort Study. Inflamm Bowel Dis. 2019;25(1):111-23.

53. Villumsen M, Aznar S, Pakkenberg B, Jess T, Brudek T. Inflammatory bowel disease increases the risk of Parkinson's disease: a Danish nationwide cohort study 1977-2014. Gut. 2019;68(1):18-24. 
54. Peter I, Dubinsky M, Bressman S, Park A, Lu C, Chen N, et al. Anti-Tumor Necrosis Factor Therapy and Incidence of Parkinson Disease Among Patients With Inflammatory Bowel Disease. JAMA Neurol. 2018;75(8):939-46.

55. Lin JC, Lin CS, Hsu CW, Lin CL, Kao CH. Association Between Parkinson's Disease and Inflammatory Bowel Disease: a Nationwide Taiwanese Retrospective Cohort Study. Inflamm Bowel Dis. 2016;22(5):1049-55.

56. Camacho-Soto A, Gross A, Searles Nielsen S, Dey N, Racette BA. Inflammatory bowel disease and risk of Parkinson's disease in Medicare beneficiaries. Parkinsonism Relat Disord. 2018;50:23-8.

57. Forsyth CB, Shannon KM, Kordower JH, Voigt RM, Shaikh M, Jaglin JA, et al. Increased intestinal permeability correlates with sigmoid mucosa alpha-synuclein staining and endotoxin exposure markers in early Parkinson's disease. PLoS One. 2011;6(12): e28032.

58. Perez-Pardo P, Dodiya HB, Engen PA, Forsyth CB, Huschens AM, Shaikh M, et al. Role of TLR4 in the gut-brain axis in Parkinson's disease: a translational study from men to mice. Gut. 2019;68(5):829-43.

59. Devos D, Lebouvier T, Lardeux B, Biraud M, Rouaud T, Pouclet $\mathrm{H}$, et al. Colonic inflammation in Parkinson's disease. Neurobiol Dis. 2013;50:42-8.

60. Pochard C, Leclair-Visonneau L, Coron E, Neunlist M, RolliDerkinderen M, Derkinderen P. Cyclooxygenase 2 is upregulated in the gastrointestinal tract in Parkinson's disease. Mov Disord. 2018;33(3):493-4.

61. Houser MC, Chang J, Factor SA, Molho ES, Zabetian CP, HillBurns EM, et al. Stool Immune Profiles Evince Gastrointestinal Inflammation in Parkinson's Disease. Mov Disord. 2018;33(5): 793-804.

62. Bessman NJ, Mathieu JRR, Renassia C, Zhou L, Fung TC, Fernandez KC, et al. Dendritic cell-derived hepcidin sequesters iron from the microbiota to promote mucosal healing. Science. 2020;368(6487):186-9.

63. McGlinchey RP, Lacy SM, Huffer KE, Tayebi N, Sidransky E, Lee JC. C-terminal alpha-synuclein truncations are linked to cysteine cathepsin activity in Parkinson's disease. J Biol Chem. 2019;294(25):9973-84

64. Schwiertz A, Spiegel J, Dillmann U, Grundmann D, Burmann J, Fassbender K, et al. Fecal markers of intestinal inflammation and intestinal permeability are elevated in Parkinson's disease. Parkinsonism Relat Disord. 2018;50:104-7.

65. Pal GD, Shaikh M, Forsyth CB, Ouyang B, Keshavarzian A, Shannon KM. Abnormal lipopolysaccharide binding protein as marker of gastrointestinal inflammation in Parkinson disease. Front Neurosci. 2015;9:306.

66. O'Donovan SM, Crowley EK, Brown JR, O'Sullivan O, O'Leary OF, Timmons S, et al. Nigral overexpression of alpha-synuclein in a rat Parkinson's disease model indicates alterations in the enteric nervous system and the gut microbiome. Neurogastroenterol Motil. 2020;32(1):e13726.

67. Chen SG, Stribinskis V, Rane MJ, Demuth DR, Gozal E, Roberts AM, et al. Exposure to the Functional Bacterial Amyloid Protein Curli Enhances Alpha-Synuclein Aggregation in Aged Fischer 344 Rats and Caenorhabditis elegans. Sci Rep. 2016;6:34477.

68. Williams-Gray CH, Wijeyekoon R, Yarnall AJ, Lawson RA, Breen DP, Evans JR, et al. Serum immune markers and disease progression in an incident Parkinson's disease cohort (ICICLEPD). Mov Disord. 2016;31(7):995-1003.

69. Tansey MG, Romero-Ramos M. Immune system responses in Parkinson's disease: Early and dynamic. Eur J Neurosci. 2019;49(3):364-83

70. Kolodziejczyk AA, Zheng D, Elinav E. Diet-microbiota interactions and personalized nutrition. Nat Rev Microbiol. 2019;17(12): $742-53$.
71. Zhernakova A, Kurilshikov A, Bonder MJ, Tigchelaar EF, Schirmer M, Vatanen T, et al. Population-based metagenomics analysis reveals markers for gut microbiome composition and diversity. Science. 2016;352(6285):565-9.

72. Yang Q, Liang Q, Balakrishnan B, Belobrajdic DP, Feng QJ, Zhang W. Role of Dietary Nutrients in the Modulation of Gut Microbiota: A Narrative Review. Nutrients. 2020;12(2).

73. Zhang $\mathrm{C}$, Zhang $\mathrm{M}$, Wang S, Han R, Cao Y, Hua W, et al. Interactions between gut microbiota, host genetics and diet relevant to development of metabolic syndromes in mice. ISME J. 2010;4(2):232-41.

74. David LA, Maurice CF, Carmody RN, Gootenberg DB, Button JE, Wolfe BE, et al. Diet rapidly and reproducibly alters the human gut microbiome. Nature. 2014;505(7484):559-63.

75. Carmody RN, Gerber GK, Luevano JM, Jr., Gatti DM, Somes L, Svenson KL, et al. Diet dominates host genotype in shaping the murine gut microbiota. Cell Host Microbe. 2015;17(1):72-84

76. Claesson MJ, Jeffery IB, Conde S, Power SE, O'Connor EM, Cusack S, et al. Gut microbiota composition correlates with diet and health in the elderly. Nature. 2012;488(7410):178-84.

77. Hills RD, Jr., Pontefract BA, Mishcon HR, Black CA, Sutton SC, Theberge CR. Gut Microbiome: Profound Implications for Diet and Disease. Nutrients. 2019;11(7).

78. Gao X, Chen H, Fung TT, Logroscino G, Schwarzschild MA, Hu FB, et al. Prospective study of dietary pattern and risk of Parkinson disease. Am J Clin Nutr. 2007;86(5):1486-94.

79. Alcalay RN, Gu Y, Mejia-Santana H, Cote L, Marder KS, Scarmeas N. The association between Mediterranean diet adherence and Parkinson's disease. Mov Disord. 2012;27(6):771-4.

80. Okubo H, Miyake Y, Sasaki S, Murakami K, Tanaka K, Fukushima W, et al. Dietary patterns and risk of Parkinson's disease: a case-control study in Japan. Eur J Neurol. 2012;19(5):6818.

81. Agarwal P, Wang Y, Buchman AS, Holland TM, Bennett DA, Morris MC. MIND Diet Associated with Reduced Incidence and Delayed Progression of ParkinsonismA in Old Age. J Nutr Health Aging. 2018;22(10):1211-5.

82. Berg D, Postuma RB, Adler CH, Bloem BR, Chan P, Dubois B, et al. MDS research criteria for prodromal Parkinson's disease. Mov Disord. 2015;30(12):1600-11.

83. Maraki MI, Yannakoulia M, Stamelou M, Stefanis L, Xiromerisiou G, Kosmidis MH, et al. Mediterranean diet adherence is related to reduced probability of prodromal Parkinson's disease. Mov Disord. 2019;34(1):48-57.

84. LaHue SC, Comella CL, Tanner CM. The best medicine? The influence of physical activity and inactivity on Parkinson's disease. Mov Disord. 2016;31(10):1444-54.

85. Marras C, Canning CG, Goldman SM. Environment, lifestyle, and Parkinson's disease: Implications for prevention in the next decade. Mov Disord. 2019;34(6):801-11.

86. Wu GD, Chen J, Hoffmann C, Bittinger K, Chen YY, Keilbaugh SA, et al. Linking long-term dietary patterns with gut microbial enterotypes. Science. 2011;334(6052):105-8.

87. Kovatcheva-Datchary P, Nilsson A, Akrami R, Lee YS, De Vadder F, Arora T, et al. Dietary Fiber-Induced Improvement in Glucose Metabolism Is Associated with Increased Abundance of Prevotella. Cell Metab. 2015;22(6):971-82.

88. Baster NT, Schmidt AW, Venkataraman A, Kim KS, Waldron C, Schmidt TM. Dynamics of Human Gut Microbiota and ShortChain Fatty Acids in Response to Dietary Interventions with Three Fermentable Fibers. mBio. 2019;10:e02566-18.

89. Desai MS, Seekatz AM, Koropatkin NM, Kamada N, Hickey CA, Wolter M, et al. A Dietary Fiber-Deprived Gut Microbiota Degrades the Colonic Mucus Barrier and Enhances Pathogen Susceptibility. Cell. 2016;167(5):1339-53 e21. 
90. Jackson A, Forsyth CB, Shaikh M, Voigt RM, Engen PA, Ramirez V, et al. Diet in Parkinson's Disease: Critical Role for the Microbiome. Front Neurol. 2019;10:1245.

91. Ghosh TS, Rampelli S, Jeffery IB, Santoro A, Neto M, Capri M, et al. Mediterranean diet intervention alters the gut microbiome in older people reducing frailty and improving health status: the NUAGE 1-year dietary intervention across five European countries. Gut. 2020.

92. Hegelmaier T, Lebbing M, Duscha A, Tomaske L, Tonges L, Holm JB, et al. Interventional Influence of the Intestinal Microbiome Through Dietary Intervention and Bowel Cleansing Might Improve Motor Symptoms in Parkinson's Disease. Cells. 2020;9(2).

93. Perez-Pardo P, Dodiya HB, Broersen LM, Douna H, van Wijk N, Lopes da Silva S, et al. Gut-brain and brain-gut axis in Parkinson's disease models: Effects of a uridine and fish oil diet. Nutr Neurosci. 2018;21(6):391-402.

94. Tieu K, Perier C, Caspersen C, Teismann P, Wu D-C, Yan S-D, et al. D- $\beta$-Hydroxybutyrate rescues mitochondrial respiration and mitigates features of Parkinson disease. Journal of Clinical Investigation. 2003;112(6):892-901.

95. VanItallie TB, Nonas C, Di Rocco A, Boyar K, Hyams K, Heymsfield SB. Treatment of Parkinson disease with dietinduced hyperketonemia: A feasibility study. Neurology. 2005;64:728-30.

96. Phillips MCL, Murtagh DKJ, Gilbertson LJ, Asztely FJS, Lynch CDP. Low-fat versus ketogenic diet in Parkinson's disease: A pilot randomized controlled trial. Mov Disord. 2018;33(8):1306-14.

97. Hernán MA, Takkouche B, Caamaño-Isorna F, Gestal-Otero JJ. A meta-analysis of coffee drinking, cigarette smoking, and the risk of Parkinson's disease. Ann Neurol. 2002;52(3):276-84.

98. Hussain SK, Dong TS, Agopian V, Pisegna JR, Durazo FA, Enayati P, et al. Dietary Protein, Fiber and Coffee Are Associated with Small Intestine Microbiome Composition and Diversity in Patients with Liver Cirrhosis. Nutrients. 2020;12(5).

99. González S, Salazar N, Ruiz-Saavedra S, Gómez-Martín M, de Los Reyes-Gavilán CG, Gueimonde M. Long-Term Coffee Consumption is Associated with Fecal Microbial Composition in Humans. Nutrients. 2020;12(5).

100. Jaquet M, Rochat I, Moulin J, Cavin C, Bibiloni R. Impact of coffee consumption on the gut microbiota: a human volunteer study. Int J Food Microbiol. 2009;130(2):117-21.

101. Boekema PJ, Samsom M, van Berge Henegouwen GP, Smout AJ. Coffee and gastrointestinal function: facts and fiction. A review. Scand J Gastroenterol Suppl. 1999;230:35-9.

102. Postuma RB, Anang J, Pelletier A, Joseph L, Moscovich M, Grimes D, et al. Caffeine as symptomatic treatment for Parkinson disease (Café-PD). Neurology. 2017;89(17):1795.

103. Takeshige-Amano H, Saiki S, Fujimaki M, Ueno S-I, Li Y, Hatano T, et al. Shared Metabolic Profile of Caffeine in Parkinsonian Disorders. Movement Disorders. 2020;35(8):143847.

104. Hill C, Guarner F, Reid G, Gibson GR, Merenstein DJ, Pot B, et al. Expert consensus document. The International Scientific Association for Probiotics and Prebiotics consensus statement on the scope and appropriate use of the term probiotic. Nat Rev Gastroenterol Hepatol. 2014;11(8):506-14.

105. Gibson GR, Scott KP, Rastall RA, Tuohy KM, Hotchkiss A, Dubert-Ferrandon A, et al. Dietary prebiotics: current status and new definition. Food Science \& Technology Bulletin: Functional Foods. 2010;7(1):1-19.

106. Varankovich NV, Nickerson MT, Korber DR. Probiotic-based strategies for therapeutic and prophylactic use against multiple gastrointestinal diseases. Front Microbiol. 2015;6:685.
107. Barichella M, Pacchetti C, Bolliri C, Cassani E, Iorio L, Pusani C, et al. Probiotics and prebiotic fiber for constipation associated with Parkinson disease: An RCT. Neurology. 2016;87(12):1274-80.

108. Yang J, Wang H-P, Zhou L, Xu C-F. Effect of dietary fiber on constipation: a meta analysis. World Journal of Gastroenterology. 2012;18(48):7378-83.

109. Dimidi E, Christodoulides S, Fragkos KC, Scott SM, Whelan K. The effect of probiotics on functional constipation in adults: a systematic review and meta-analysis of randomized controlled trials. Am J Clin Nutr. 2014;100(4):1075-84

110. Dimidi E, Christodoulides S, Scott SM, Whelan K. Mechanisms of Action of Probiotics and the Gastrointestinal Microbiota on Gut Motility and Constipation. Adv Nutr. 2017;8(3):484-94.

111. Markowiak P, Slizewska K. Effects of Probiotics, Prebiotics, and Synbiotics on Human Health. Nutrients. 2017;9(9).

112. Srivastav S, Neupane S, Bhurtel S, Katila N, Maharjan S, Choi H, et al. Probiotics mixture increases butyrate, and subsequently rescues the nigral dopaminergic neurons from MPTP and rotenoneinduced neurotoxicity. J Nutr Biochem. 2019;69:73-86.

113. Magistrelli L, Amoruso A, Mogna L, Graziano T, Cantello R, Pane M, et al. Probiotics May Have Beneficial Effects in Parkinson's Disease: In vitro Evidence. Front Immunol. 2019;10: 969.

114. Hsieh TH, Kuo CW, Hsieh KH, Shieh MJ, Peng CW, Chen YC, et al. Probiotics Alleviate the Progressive Deterioration of Motor Functions in a Mouse Model of Parkinson's Disease. Brain Sci. 2020;10(4).

115. Castelli V, d'Angelo M, Lombardi F, Alfonsetti M, Antonosante A, Catanesi M, et al. Effects of the probiotic formulation SLAB51 in in vitro and in vivo Parkinson's disease models. Aging (Albany NY). 2020;12(5):4641-59.

116. Goya ME, Xue F, Sampedro-Torres-Quevedo C, Arnaouteli S, Riquelme-Dominguez L, Romanowski A, et al. Probiotic Bacillus subtilis Protects against alpha-Synuclein Aggregation in C. elegans. Cell Rep. 2020;30(2):367-80 e7.

117. Tamtaji OR, Taghizadeh M, Daneshvar Kakhaki R, Kouchaki E, Bahmani F, Borzabadi S, et al. Clinical and metabolic response to probiotic administration in people with Parkinson's disease: A randomized, double-blind, placebo-controlled trial. Clin Nutr. 2019;38(3):1031-5.

118. Bartlett JG, Chang TW, Gurwith M, Gorbach SL, Onderdonk AB. Antibiotic Associated Pseudomembranous Colitis due to ToxinProducing Clostridia. NEJM. 1978;298(10):531-4.

119. McFarland LV. Epidemiology, Risk Factors and Treatments for Antibiotic-Associated Diarrhea. Dig Dis. 1998;16(292-307).

120. Ianiro G, Tilg H, Gasbarrini A. Antibiotics as deep modulators of gut microbiota: between good and evil. Gut. 2016;65(11):190615.

121. Sullivan $\AA$, Edlund C, Nord CE. Effect of antimicrobial agents on the ecological balance of human microflora. The Lancet Infectious Diseases. 2001;1(2):101-14.

122. Jernberg C, Lofmark S, Edlund C, Jansson JK. Long-term impacts of antibiotic exposure on the human intestinal microbiota. Microbiology. 2010;156(Pt 11):3216-23.

123. Mertsalmi TH, Pekkonen E, Scheperjans F. Antibiotic exposure and risk of Parkinson's disease in Finland: A nationwide casecontrol study. Mov Disord. 2020;35(3):431-42.

124. Palacios N, O'Reilly EJ, Schwarzschild MA, Ascherio A. LongTerm Use of Antibiotics and Risk of Parkinson's Disease in the Nurses' Health Study. Parkinsons Dis. 2020;2020:4038375.

125. Pierantozzi M, Pietroiusti A, Brusa L, Galati S, Stefani A, Lunardi $\mathrm{G}$, et al. Helicobacter pylori eradication and 1-dopa absorption in patients with PD and motor fluctuations. Neurology. 2006;66(12): 1824-9. 
126. Tan AH, Lim SY, Mahadeva S, Loke MF, Tan JY, Ang BH, et al. Helicobacter pylori Eradication in Parkinson's Disease: A Randomized Placebo-Controlled Trial. Mov Disord. 2020

127. Fasano A, Bove F, Gabrielli M, Petracca M, Zocco MA, Ragazzoni E, et al. The role of small intestinal bacterial overgrowth in Parkinson's disease. Mov Disord. 2013;28(9):1241-9.

128. Ponziani FR, Scaldaferri F, Petito V, Paroni Sterbini F, Pecere S, Lopetuso LR, et al. The Role of Antibiotics in Gut Microbiota Modulation: The Eubiotic Effects of Rifaximin. Dig Dis. 2016;34(3):269-78.

129. Du Y, Ma Z, Lin S, Dodel RC, Gao F, Bales KR, et al. Minocycline prevents nigrostriatal dopaminergic neurodegeneration in the MPTP model of Parkinson's disease. Proc Natl Acad Sci U S A. 2001;98(25):14669-74.

130. Investigators NN-P. A pilot clinical trial of creatine and minocycline in early Parkinson disease: 18-month results. Clin Neuropharmacol. 2008;31(3):141-50.

131. Elewa HF, Hilali H, Hess DC, Machado LS, Fagan SC. Minocycline for short-term neuroprotection. Pharmacotherapy. 2006;26(4):515-21.

132. Gupta A, Saha S, Khanna S. Therapies to modulate gut microbiota: Past, present and future. World J Gastroenterol. 2020;26(8): $777-88$.

133. Allegretti JR, Mullish BH, B.Chir MB, Kelly C, Fischer M. The Evolution of the use of Faecal Microbiota Transplantation and Emerging Therapeutic Indications. Lancet. 2019;10196:420-31.

134. Zhang Z, Mocanu V, Cai C, Dang J, Slater L, Deehan EC, et al. Impact of Fecal Microbiota Transplantation on Obesity and Metabolic Syndrome-A Systematic Review. Nutrients. 2019;11(10)

135. Allegretti JR, Kassam Z, Mullish BH, Chiang A, Carrellas M, Hurtado J, et al. Effects of Fecal Microbiota Transplantation With Oral Capsules in Obese Patients. Clin Gastroenterol Hepatol. 2020;18(4):855-63.e2.

136. Vendrik KEW, Ooijevaar RE, de Jong PRC, Laman JD, van Oosten BW, van Hilten JJ, et al. Fecal Microbiota Transplantation in Neurological Disorders. Front Cell Infect Microbiol. 2020;10:98.

137. Sun MF, Zhu YL, Zhou ZL, Jia XB, Xu YD, Yang Q, et al. Neuroprotective effects of fecal microbiota transplantation on MPTP-induced Parkinson's disease mice: Gut microbiota, glial reaction and TLR4/TNF-alpha signaling pathway. Brain Behav Immun. 2018;70:48-60.
138. Huang H, Xu H, Luo Q, He J, Li M, Chen H, et al. Fecal microbiota transplantation to treat Parkinson's disease with constipation: A case report. Medicine (Baltimore). 2019;98(26):e16163.

139. Xue LJ, Yang XZ, Tong Q, Shen P, Ma SJ, Wu SN, et al. Fecal microbiota transplantation therapy for Parkinson's disease: A preliminary study. Medicine (Baltimore). 2020;99(35):e22035.

140. Craven LJ, Nair Parvathy S, Tat-Ko J, Burton JP, Silverman MS. Extended Screening Costs Associated With Selecting Donors for Fecal Microbiota Transplantation for Treatment of Metabolic Syndrome-Associated Diseases. Open Forum Infect Dis. 2017;4(4):ofx243.

141. Sandler M, Goodwin BL, Ruthven CRJ, Calne DB. Therapeutic Implications in Parkinsonism of m-Tyramine Formation from LDopa in Man. Nature. 1970;229(5284):414-5.

142. Goldin BR, Peppercorn MA, Goldman P. Contributions of Host and Intestinal Micro-flora in the Metabolism of L-DOPA by the Rat. J Pharmacol Exp Ther. 1973;186(1):160-6.

143. van Kessel SP, El Aidy S. Contributions of Gut Bacteria and Diet to Drug Pharmacokinetics in the Treatment of Parkinson's Disease. Front Neurol. 2019;10:1087.

144. Maini Rekdal V, Bess EN, Bisanz JE, Turnbaugh PJ, Balskus EP. Discovery and inhibition of an interspecies gut bacterial pathway for Levodopa metabolism. Science. 2019;364(6445).

145. Dou J, Bennett MR. Synthetic Biology and the Gut Microbiome. Biotechnol J. 2018;13(5):e1700159.

146. Zhou Z, Chen X, Sheng H, Shen X, Sun X, Yan Y, et al. Engineering probiotics as living diagnostics and therapeutics for improving human health. Microb Cell Fact. 2020;19(1):56.

147. Isabella VM, Ha BN, Castillo MJ, Lubkowicz DJ, Rowe SE, Millet YA, et al. Development of a synthetic live bacterial therapeutic for the human metabolic disease phenylketonuria. Nat Biotechnol. 2018;36(9):857-64.

148. Group TPS. A randomized trial of relamorelin for constipation in Parkinson's disease (MOVE-PD): Trial results and lessons learned. Parkinsonism Relat Disord. 2017;37:101-5.

149. Maetzler W, Liepelt I, Berg D. Progression of Parkinson's disease in the clinical phase: potential markers. Lancet Neurol. 2009;8(12):1158-71.

Publisher's Note Springer Nature remains neutral with regard to jurisdictional claims in published maps and institutional affiliations. 